\title{
Alfabetización climática en la formación inicial y continua de docentes
}

\section{Climate literacy in initial and continuing teacher training}

\author{
Marisol Lopera Pérez* \\ (D) https://orcid.org/0000-0002-2548-6516 \\ Sara Villagrá Sobrino** \\ (iD https://orcid.org/0000-0003-2516-0492
}

Tipo de Artículo: Reportes de investigación y ensayos inéditos

Doi: 10.17533/udea.unipluri.20.1.05

Cómo citar este artículo:

Lopera Pérez, M., y Villagrá Sobrino, S. (2020). Alfabetización climática en la formación

inicial y continua de docentes. Uni-Pluriversidad, 20(1), e2020104. doi:10.17533/udea.unipluri.20.1.05

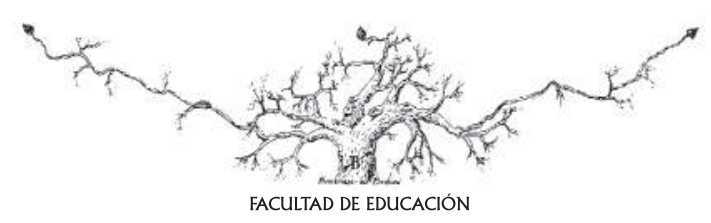

Recibido: 2020-02-05 • Aprobado: 2020-07-12

* Universidad de Antioquia. Colombia

E-mail: marisol.loperap@udea.edu.co

** Universidad de Valladolid. España.

E-mail: sarena@pdg.uva.es 


\title{
Resumen
}

El cambio climático es un asunto relevante en la actualidad, puesto que en todos los contextos se considera ineludible responder a las emergentes tensiones y problemáticas asociadas. Así que, esta investigación educativa derivada de una tesis doctoral, está fundamentada en la alfabetización climática de docentes en formación inicial y continua, a través del diseño e iteración de un modelo, desde donde se llevaron a cabo experiencias de formación en la Universidad de Valladolid (España) y a través del Centro de Formación del Profesorado e Innovación Educativa (CFIE, Valladolid). El proceso se entiende como una investigación basada en diseño, la cual tuvo como punto de partida la revisión sistemática de la literatura que permitió el diseño de un nuevo modelo y su posterior iteración. Los resultados están enmarcados en dos categorías de análisis: alfabetización climática y profesionalización docente. En relación con la primera, se destaca que los hallazgos visibilizan los conocimientos que los participantes tenían sobre el cambio climático y su reestructuración a partir de la conexión entre diferentes áreas. Además, se valoran las actitudes emocionales, cognoscitivas y conductuales. Con respecto a la profesionalización docente, los resultados dan cuenta de una aproximación a diferentes estrategias de enseñanza y aprendizaje susceptibles de aplicar en su práctica como docentes.

Palabras clave: alfabetización climática, profesionalización docente, investigación basada en diseño, educación ambiental, experiencias de formación.

\begin{abstract}
Climate change is an issue of a great relevance today, since in all contexts it is considered unavoidable to respond to the emerging tensions and associated problems. Therefore, this research is focused on the climate literacy of preservice and inservice teachers, through the design and iteration of a model that favors the development of training experiences (at the University of Valladolid and through the Center of Teacher Training and Educational Innovation-CFIE, Valladolid-). The process is understood as a design-based research, which had as a starting point a systematic review of the literature that allowed to design a new model and its iteration. Results are framed in two categories: environmental literacy and teacher professionalization. In relation to the first one, findings make visible the knowledge that the participants had about climate change and its restructuring based on the connection between different areas. Likewise, emotional, cognitive, and behavioral attitudes of the participants were described. Regarding teacher professionalization, results show an approach to different teaching and learning strategies that can be applied in teaching practice.
\end{abstract}

Keywords: climate literacy, training teachers, design-based research, environmental education, training teacher experiences. 


\section{INTRODUCCIÓN}

La Educación Ambiental (en adelante, EA) se gestó en la década de los años 70 , como resultado de la movilización social (civil y estudiantil), política y cultural que buscaba visibilizar el riesgo que corría la sociedad postmoderna, bajo la dinámica generada por la racionalidad economicista cartesiana y los modelos de producción. Fue así como las reflexiones del Club de Roma (1968) y la Cumbre de Estocolmo (1972) cimentaron el camino para que en Tbilisi (1977) se planteara la necesidad de transformar los sistemas educativos reenfocándolos hacia una nueva ética, basada en la equidad, la justicia social y ambiental.

En el 2015, la Asamblea General de la ONU propuso la Agenda 2030 para el Desarrollo Sostenible, como un plan para favorecer a las personas y la biosfera, además, con la intención de fortalecer la paz universal. La Agenda plantea 17 Objetivos para el Desarrollo Sostenible (ODS) con 169 metas de carácter integrado e indivisible que abarcan las esferas económica, social y ambiental. Esta directriz internacional ha permeado lo educativo y ha sugerido el direccionamiento de los procesos formativos que permitan proponer estrategias participativas, colaborativas y sinérgicas en contextos locales y regionales, sin perder de vista lo global.

Particularmente, en el contexto espanol se ha hecho un esfuerzo por favorecer la ambientalización curricular en todos los niveles educativos. En especial, se destaca el interés por un asunto sociocientífico como el cambio climático, una realidad inexorable, que afecta a todas las personas y a la mayoría de los contextos. En la literatura, se hace referencia a la alfabetización climática o educación para el cambio climático como un aspecto determinante en la formación de docentes, puesto que, como lo mencionan Plutzer et al. (2016), el profesorado no recibe en todos los casos una formación pertinente para abordar estos temas en el aula; por esta razón pueden tener algunas confusiones conceptuales y dificultad para plantear estrategias pedagógicas.

En esta investigación se entiende que la alfabetización climática -especialmente en la formación de docentes- provee los conocimientos -desde una perspectiva transdisciplinar- necesarios para entender el sistema climático en interacción con los sistemas socio culturales, desde posicionamientos críticos que lean las determinaciones políticas y económicas que están acelerando los efectos de manera sistémica. Así mismo, el concepto de alfabetización climática se vincula con las actitudes -emocionales/afectivas, cognoscitivas y conductuales- y con la generación de ideas que posibilitan reflejar dicha alfabetización en realidad educativa, como mecanismo de adaptación y transformación.

En consecuencia, este artículo reporta los resultados que se obtuvieron en tres ex- 
periencias de formación de docentes (inicial y continua) sobre el cambio climático. Estas experiencias hicieron parte de una investigación doctoral planteada desde la perspectiva metodológica de la investigación basada en diseño (Reeves, Herrington \& Oliver, 2005;
Wang \& Hannafin, 2005). De tal manera que el objetivo general de esta investigación es analizar la alfabetización climática y profesionalización docente en procesos de formación a través de la aplicación de un modelo didáctico.

\section{REFERENTES CONCEPTUALES}

\section{Alfabetización climática}

El cambio climático es uno de los asuntos más relevantes en la actualidad, de hecho, múltiples fenómenos naturales y socio culturales están asociados con este. Así que es latente la necesidad de ofrecer una educación que permita formar y aportar a la resolución de los problemas asociados, o lo que se denomina alfabetización climática (Nayan, Mahat, Hashim, Saleh \& Norkhaidi, 2018; Niepold, Herring \& McConville, 2007).

Se espera que las personas que están alfabetizadas en lo relativo al cambio climático tengan un pensamiento sistémico y crítico, sepan cómo encontrar información científica veraz, discutan de manera significativa los problemas, sean capaces de tomar decisiones responsables y prudentes con respecto a las acciones que afectan el clima. Así mismo, se espera que, a través de un proceso de alfabetización climática, las personas sean conscientes de la relación básica entre el clima y la vida humana y que puedan evaluar la validez de un argumento respecto a este tema.

Ahora bien, según lo mencionan Gaudiano \& Cartea (2020), se debe tener claridad entre formar sobre el clima o educar para el cambio, además, desde los procesos educativos es preciso ahondar en algunos asuntos: la sensibilidad por lo local, desde estrategias pedagógicas basadas en el contexto o situadas; la participación ciudadana hacia cuestiones de gobernanza y gestión comunitaria del riesgo; la generación de proyectos o investigaciones en todos los niveles escolares, que respondan a las inquietudes y necesidades de los estudiantes y sus comunidades; y la superación de posiciones fatalistas y emociones negativas sobre la actual situación.

Desde otras perspectivas, la alfabetización climática está fuertemente vinculada con otras alfabetizaciones: científica, ecológica, oceánica y energética (Azevedo \& Marques, 2017). Particularmente, tiene una relación explícita con el ODS 13 -Acción por el clima: Adoptar medidas urgentes para combatir el cambio climático y sus efectos-, e implícita con los ODS 4, 16 y 17.

\section{Profesionalización docente}

El tema de la profesionalización docente en relación con el cambio climático ha sido estudiado por Goldman, Yavetz \& Pe'er (2006), quienes enfatizan en su importancia para la formación de las generaciones futuras, así que, su adecuada preparación a través de los diferentes programas de formación inicial resulta determinante. Asimismo, la UNESCO (1997) identificó una 
preparación insuficiente de los docentes en este tema, lo cual se refleja en una implementación inadecuada de la educación ambiental en los centros educativos. Por esta razón, Pérez-Rodríguez, Varela-Losada, Lorenzo-Rial y Vega-Marcote (2017) dejan en evidencia que la formación inicial y continua de docentes debe favorecer la alfabetización climática para garantizar el desarrollo de capacidades, la participación y la innovación.

En particular, la profesionalización docente "es el proceso que deviene del desarrollo social, económico y laboral y que como tendencia es deseable porque garantiza mayor calidad en el desempeño profesional" (Tejada-Fernández, 2009, p. 465). En este sentido, involucra una amplia gama de conocimientos y/o habilidades que le posibilitan al docente, en el presente o futuro quehacer, alcanzar un desempeño satisfactorio que le posibilite maniobrar o gestionar las múltiples dificultades que se puede encontrar en la realidad de aula y en los centros educativos.

Se resaltan dos tipos de conocimiento en los procesos de profesionalización docente: el curricular y el didáctico. En primera instancia, el conocimiento curricular en simbiosis con la EA es de absoluta relevancia, puesto que requiere una profunda contextualización por parte de los centros educativos, en la mayoría de los casos en cabeza de los docentes. Incluso, algunas preguntas sobre el medio ambiente y, en particular, sobre el cambio climático rara vez se incluyen en el currículo, pero están relacionadas con la naturaleza y, de forma específica, con la interacción ser humano-ambiente, cultura-naturaleza (Payne, 2006). En sintonía con lo anterior, el currículo debe ser un elemento del sistema educativo que posibilite la apertura para el redescubrimiento de cómo se interactúa con el medio ambiente desde relaciones horizontales con el otro y lo otro. El planteamiento curricular en términos de principios siempre ha sido un desafío para la EA.

En segunda instancia, lo didáctico se relaciona con el Pedagogical Content Knowledge $(P C K)$ o Conocimiento Didáctico del Contenido, que hace referencia al conocimiento de diferentes estrategias didácticas, enfoques y mecanismos de secuenciación de actividades que pueden ser utilizadas (Gess-Newsome, 2015). Para Shulman (1986, p. 9) este tipo de conocimiento se refiere a "los tópicos que más regularmente se enseñan en un área, las formas más útiles de representación de las ideas, las analogías más poderosas, ilustraciones, ejemplos, explicaciones y demostraciones, $\mathrm{y}$, en una palabra, la forma de representar y formular la materia". Por su parte, Bolívar (2005), desde sus profundas reflexiones sobre el conocimiento de los docentes, considera que entre los componentes del Conocimiento Didáctico del Contenido se han resaltado las concepciones, valores y creencias de lo que significa enseñar una determinada materia en un determinado nivel y contexto.

\section{Metodología}

La investigación doctoral de donde se deriva este trabajo se vincula con la perspectiva metodológica de investigación ba- sada en diseño (Reeves, Herrington \& Oliver, 2005; Wang \& Hannafin, 2005), puesto que es relevante para solucionar problemas 
educativos reales y avanzar hacia consolidaciones teóricas e, incluso, abordar retos educativos de alto nivel. Además, posibilita la transdisciplinariedad, el uso de diversos métodos y la inclusión de diferentes participantes.

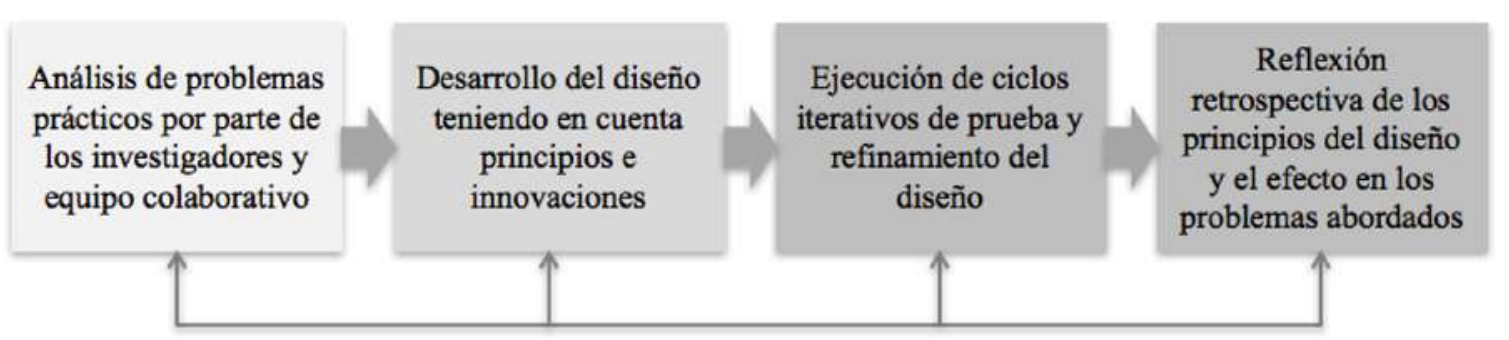

Como se presenta en la Figura 1, esta metodología de investigación se adaptó a partir de las etapas genéricas de diseño, iteración y reflexión.

Figura 1. Dinámica de problematización, planteamiento de soluciones, aplicación de métodos y principios de diseño. Adaptado de Amiel y Reeves (2008).

El diseño se realizó posterior a una revisión de literatura que permitió identificar los elementos -procedimientos- que se debía considerar para las experiencias de formación de docentes. Como se presenta en la
Figura 2, la formación se realizó a partir de 3 fases: dirigida, mediadora y semidirigida colaborativa. Cada una de estas fases se describe por una serie de actividades.

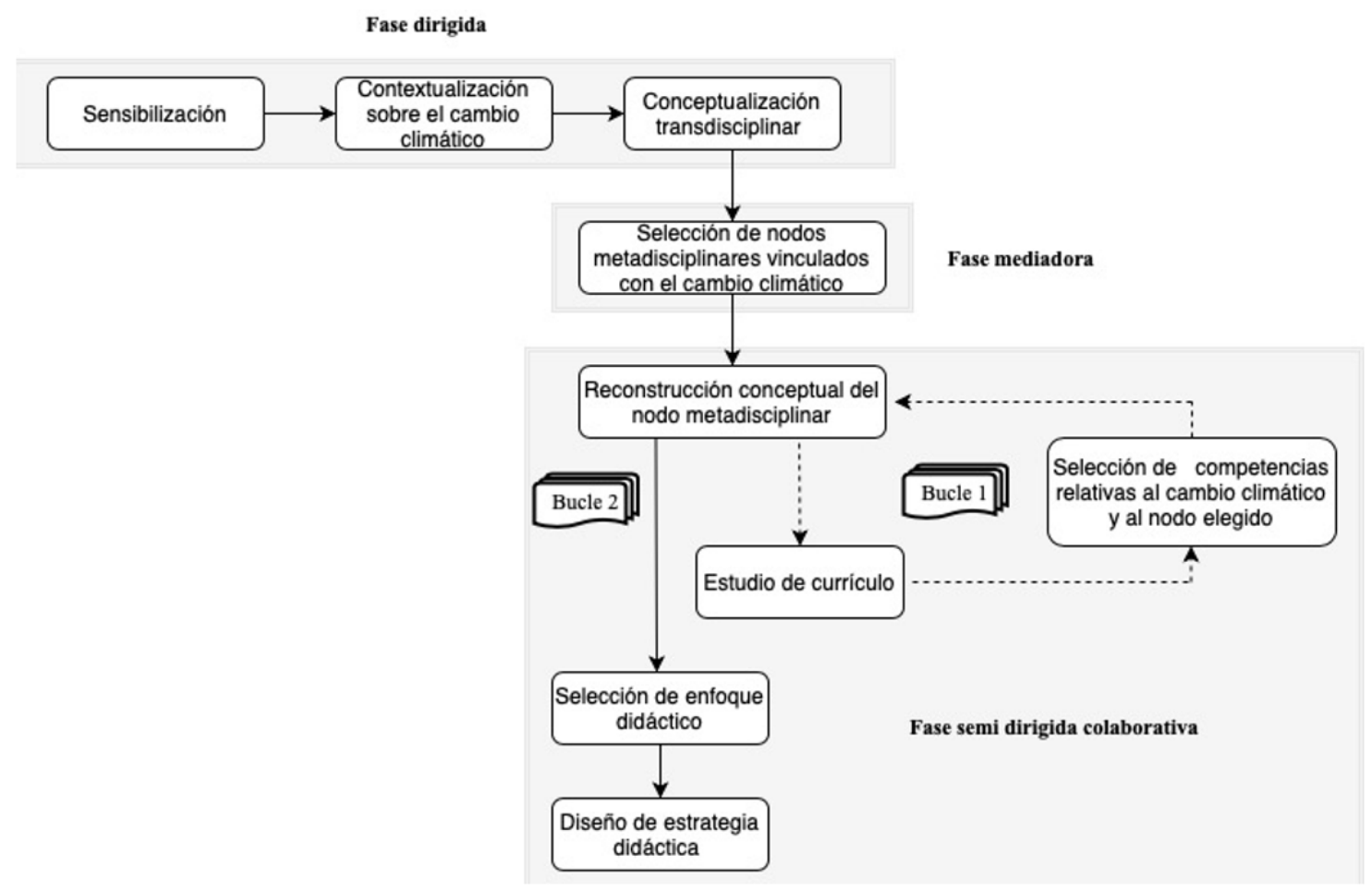

Figura 2. Estructura genérica de modelo de formación inicial y continua de docentes. Tomada de Lopera-Pérez (2019). 
En primer lugar, la fase dirigida buscaba que los docentes realizaran una contextualización del cambio climático (geográfica y política), así como una conceptualización general a partir de la integración de diferentes áreas de conocimiento (Ciencias Experimentales, Ciencias Sociales, Ética, Ciencias Políticas, entre otras). En segundo lugar, la fase mediadora se caracteriza por un estrecho diálogo entre los agentes implicados (formadora, docentes en formación) para la elaboración de una reconstrucción conceptual más profunda, a través de la selección de un concepto/nodo metadisciplinar significativo (la energía, el agua, lluvia ácida, acidificación de los océanos). En este punto, se inició el trabajo colaborativo entre los docentes en formación inicial o continua.

En último lugar, en la fase semidirigida colaborativa, los docentes en formación leen y estudian en equipos el currículo (primaria, secundaria y/o bachillerato), ubican el nodo metadisciplinar, identifican los contenidos relativos a este nodo y reorganizan su estructura conceptual (bucle reflexivo 1: disciplinar-curricular); en adición, encaminan esta reestructuración hacia el diseño de una estrategia didáctica que se puede aplicar en los centros educativos "proyección" (bucle reflexivo 2: curricular-didáctico). Las estrategias didácticas tenían diferentes enfoques: simulaciones (real data), modelización sobre el cambio climático, aprendizaje por descubrimiento, aprendizaje por indagación, aprendizaje basado en proyectos, outdoor activities, TIC para la enseñanza del cambio climático.

Posteriormente, el diseño se iteró en tres Experiencias de Formación de docentes (en adelante, EF). En la $\mathrm{EF}_{1}$ participaron 12 docentes en formación inicial de la Universi- dad de Valladolid (Grado de Educación Primaria). En la $\mathrm{EF}_{2}$ participaron 18 docentes de Ciencias Experimentales en formación inicial (Máster en profesorado de Secundaria, modalidades Física/Química, Biología/ Geología). Finalmente, la $\mathrm{EF}_{3}$ se realizó con 9 docentes en ejercicio de la ciudad de Valladolid, a través del Centro de Formación del Profesorado e Innovación Educativa (CFIE, Valladolid).

Durante la aplicación del diseño se utilizó una estrategia mixta para la recolección y análisis de datos. En particular, los datos de naturaleza cuantitativa se recogieron mediante cuestionarios -inicial y final- (39), a los cuales se les realizó un análisis estadístico descriptivo. Los datos cualitativos se obtuvieron a través de transcripciones de audios (15), del análisis de diversos documentos generados por los informantes como mapas conceptuales (9) y estrategias didácticas (17). A estos documentos se les aplicó el análisis de contenido asistido por ordenador (Atlas.ti, v. 8.0), como "técnica de investigación destinada a formular, a partir de ciertos datos, inferencias reproducibles y válidas que puedan aplicarse a su contexto" (Krippendorff, 2004, p. 28).

El análisis de los datos -cuantitativos y cualitativos- estuvo vinculado a dos categorías de análisis. La primera fue la alfabetización climática descrita por dos subcategorías: conocimientos sobre el cambio climático y actitudes. La segunda categoría fue la profesionalización docente descrita por dos subcategorías: conocimiento curricular y conocimiento didáctico del contenido. Los resultados se expondrán desde la perspectiva de dichas categorías. 


\section{RESULTADOS Y DISCUSIÓN}

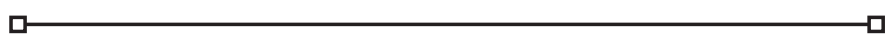

Alfabetización climática: conocimientos y actitudes sobre el cambio climático

El primer aspecto relacionado con los conocimientos sobre el cambio climático son las concepciones o corrientes de pensamiento dominantes en el discurso de los participantes y se relacionan con las reportadas por diferentes autores. Estas concepciones se exponen a continuación.

La red semántica que se presenta en la Figura 3 visibiliza la corriente naturalista latente en el discurso de algunos participan- tes, así que los códigos de los bloques grises coinciden con las primeras definiciones y representaciones científicas sobre el clima que elaboraron Landsberg (1981) y Köpen (1948), donde se le considera como condición física relativa a la atmósfera en términos locales y regionales, lo cual se relaciona con la corriente biorregionalista (citas 5:3 y 5:1) y cientificista. Se aclara que no se identificaron concepciones que involucraran el pensamiento sistémico que concibieran a través de las interacciones entre los sistemas climáticos.

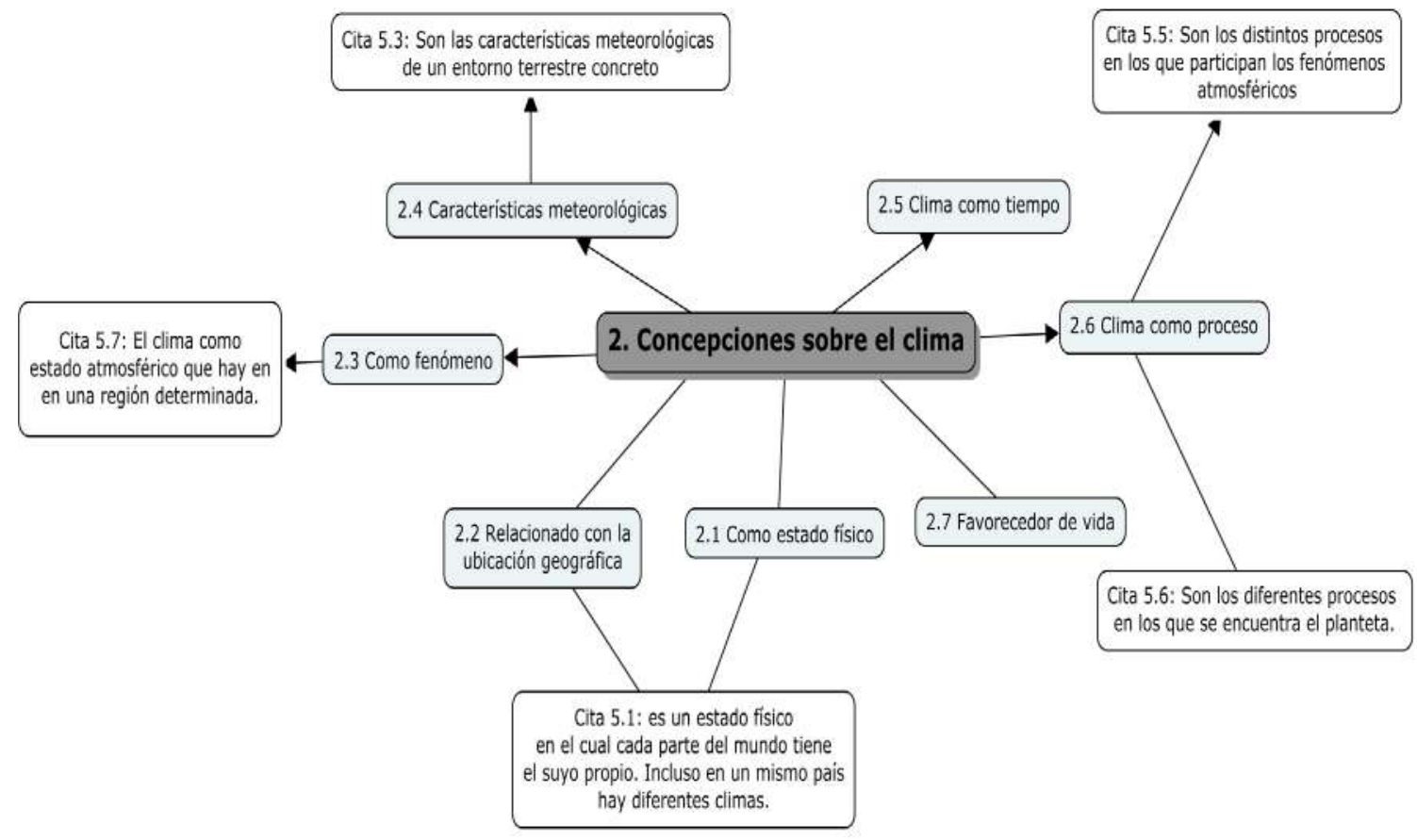

Figura 3. Red semántica de las diferentes concepciones de los docentes en formación acerca del clima.

Por otra parte, en las tres EF se identificó la corriente de pensamiento que se asociaba al principio de la sostenibilidad, puesto que los participantes reconocieron que es un término comúnmente utilizado y apropiado por los medios de comunicación. En parti- cular, para algunos docentes en servicio en la $\mathrm{EF}_{3}$, las nociones sobre desarrollo sostenible están presentes en diferentes escenarios: la política, la economía, el ámbito social y educativo. 
DF32: La definición de los medios es el tipo de desarrollo que garantiza que las generaciones actuales puedan mantener su calidad de vida y no pongan en peligro las generaciones futuras y va en contra vía del modelo económico. Un planeta finito y un modelo de explotación ilimitado no es compatible.

DF37: Hay una cosa que es importante y es que todo esto lleva a los chavales a pensar en la economía y en que hacen falta nuevos modelos económicos; esta idea tan liberadora de cambiar la economía del Carbono por la economía del sol. Cuando hablas de esto con los alumnos, si logras articularlo bien, ves que esa idea gusta y eso es hablar de democracia, también es hablar de libertad, que ahora desgraciadamente parece que no existieran.

$\left(\mathrm{EF}_{3}\right.$ fase dirigida. Transcripción de audio. 17/02/2016)

En sintonía con lo anterior, durante una de las conversaciones grupales en la $\mathrm{EF}_{2}$ se retomó el tema del desarrollo sostenible, considerando que es un concepto condicionado por el modelo económico dominante, donde se entiende la naturaleza y los recursos naturales como una externalidad susceptible de internalizar con impuestos y sanciones.

DF13: Sí que es necesario el pago de impuestos para remediar. Lo que pasa es que el dinero debería ser suficiente para pagar todos los gastos que supone restaurar o para evitar.

DF18: El problema no es tanto la cantidad de dinero, sino a dónde va, quién lo gestiona. Por ejemplo, con la presa que se rompió en Brasil y vertió cantidades enormes de residuos mineros en el bosque amazónico, le aseguro que el dinero que pagaron las multinacionales se fue al bolsillo de alguien.

DF27: El desarrollo sostenible es como vamos a hacer esto a gran escala y a dar soluciones a gran escala, que lue- go no son reales para dejar la conciencia tranquila. Por ejemplo, he derramado tantas toneladas de petróleo, pero he pagado no sé cuánto.

$\left(\mathrm{EF}_{2}\right.$ fase dirigida. Transcripción de audio. 26/04/2016)

La interacción entre los participantes evidencia la necesidad de que los principios del desarrollo sostenible se aborden y entiendan de forma holística y crítica por los docentes, antes de apropiarlos e incorporarlos en el currículo y en las propuestas didácticas. Esto es coherente con lo propuesto por Sauvé (1996; 2005), quien considera que más que una educación enfocada en el desarrollo sostenible/sustentable, se debería apuntar a la consolidación de la educación para el desarrollo de sociedades responsables, pues este enfoque daría a la EA un objetivo más rico.

En síntesis, las evidencias presentadas dan cuenta de diversas concepciones presentes en el discurso de los participantes. En primer lugar, la concepción que se relaciona con la identificación de componentes medio ambientales reportada previamente por Astolfi (1998). En segundo lugar, concepciones ecocéntricas que reflejan la valoración de la interacción naturaleza-sociedad, coherentes con lo mencionado por Ballantyne (1995). En tercer lugar, concepciones reportadas desde la cartografía de Sauvé (2005), como son la naturalista, cientificista, moral ética, enmarcadas en el desarrollo sostenible y resolutiva.

Ahora bien, en relación con conocimientos previos sobre el cambio climático, los participantes en la $\mathrm{EF}_{1}$, como se presenta en la Tabla 1, dieron respuestas a los ítems planteados que dan cuenta de posicionamientos y niveles de conceptualización heterogéneos. 
Tabla 1.

Respuestas de los participantes de la EF, en algunos items del cuestionario inicial.

\begin{tabular}{ll}
\hline \multicolumn{1}{c}{ Ítems abiertos } & \multicolumn{1}{c}{ Respuestas* $^{*}$} \\
\hline ¿Cuáles son las causas del cambio climático? & $\begin{array}{l}\text { Desaparición de la capa de ozono, aumento de } \\
\text { los gases efecto invernadero y uso de combus- } \\
\text { tibles fósiles. }\end{array}$ \\
\hline $\begin{array}{l}\text { ¿Cuáles son las consecuencias del cambio } \\
\text { climático? En orden de importancia. }\end{array}$ & $\begin{array}{l}\text { Desaparición/descongelamiento de los casque- } \\
\text { tes polares y glaciares, aumento del nivel del } \\
\text { mar, calentamiento global, pérdida de biodiver- } \\
\text { sidad. }\end{array}$ \\
\hline ¿Cuáles son los gases que aportan al efecto \\
invernadero y calentamiento global?
\end{tabular}

*Se ubican todas las respuestas que los participantes dieron a los ítems.

En este sentido, se destaca que inicialmente no incluyeron el cambio climático como uno de los problemas ambientales relevantes. Así mismo, se evidencia resistencia para diferenciar causas antrópicas y causas relacionadas con las características y dinámicas planetarias, centrándose principalmente en consecuencias de corte bio-físico. Ahora bien, en contraste con lo anterior, algunos docentes en formación inicial, al finalizar la $\mathrm{EF}_{1}$, expusieron argumentos más elaborados que los iniciales, puesto que integraron elementos explicativos de mayor complejidad que implicaban lo biológico, fisicoquímico, social, cultural, económico y por supuesto político.

DF4: Uno de los grandes causantes de la crisis planetaria es el Hommo Sapiens, ya que cambia el mundo, conquista los medios, en definitiva, se apodera de los territorios.

DF7: Pérdida de biodiversidad de numerosos ecosistemas y pérdida de especies, cambio del hombre con respecto a la naturaleza por sobre explotación. El deshielo de numerosos glaciares.

DF5: Agotamiento de los recursos pesqueros, el calentamiento global por las emisiones de $\mathrm{CO}_{2}$ debido al consu- mo de materiales fósiles como medio de obtención de energía, un $50 \%$ de las plantaciones se dedican a la generación de biodiesel o cereales para producción de carne.

DF6: La educación es escasa, la esperanza de vida es corta, muchas poblaciones viven en extrema pobreza.

$\left(\mathrm{EF}_{1}\right.$, fase dirigida. Cuestionario final. Enero de 2015)

Por otro lado, el discurso de los participantes en la $\mathrm{EF}_{2-3}$ dejó en evidencia algunos impactos de los medios de comunicación en la divulgación de los problemas asociados al cambio climático. Además, en la superposición de lo local sobre lo global y, en ocasiones, en la generación de imaginarios que no son coherentes con las realidades socio ambientales.

DF21: Entonces, claro, es un problema, porque además las emisiones en China, yo la última vez que vi las noticias, la gente iba con mascarillas porque es que estaban en una situación de alerta roja, totalmente. Al final se llega a tomar decisiones ya drásticas en el sentido de todos tenemos que hacer lo mismo o por más que unos pocos hagan y no lo consiguen, pues no avanzamos. 
$\left(\mathrm{EF}_{2}\right.$, fase dirigida. Transcripción de audio. 21/01/2016)

Estos hallazgos dejan ver que para algunos docentes la divulgación de los asuntos ambientales está permeada por intereses y presiones de todo tipo. Además, expresaron posicionamientos críticos y reflexivos en torno a la participación política de las diferentes naciones para lograr reducciones significativas en las emisiones de $\mathrm{CO}_{2}$, para la disminución de la deforestación a nivel global y en torno a potenciar la generación de energías limpias, asunto sensible en el contexto español. En consecuencia, las discusiones vislumbraron la necesidad de asumir la corresponsabilidad de los acuerdos a los que se llega sobre temas ambientales, a pesar de que generan preocupación o desesperanza en algunos momentos.

DF35: Yo tiendo a ser positiva y hay un acuerdo en que es necesario mayor esfuerzo, pero hay que destacar que hay mayor consenso entre los países en vía de desarrollo y los desarrollados.

DF34: Luego de ver tantos años en los que las conferencias no llegaban a nada, era bastante desolador, al menos se tiene un texto para poder empezar, sin embargo, una cosa es decir que se va a poner un poco de freno a la cosa y otra ¿cuánto va a costar?, ¿es vinculante o no vinculante como se van a hacer las cosas?

$\left(\mathrm{EF}_{3}\right.$, fase dirigida. Transcripción de audio. 17/02/2016)

Con respecto al posicionamiento político, se notó una activa participación sobre todo en los momentos de discusión, desde donde se favoreció el diálogo, la discusión, la manifestación de emociones, opiniones y quejas e insatisfacciones personales y profesionales de los docentes en formación.

\section{La estructuración de conocimientos mediante el mapeo conceptual}

La estructuración o reestructuración del conocimiento sobre el cambio climático se realizó a través del uso de los mapas conceptuales como herramientas metacognitivas. De tal manera que, los 39 docentes -en formación inicial y continua- implicados en estas tres experiencias, construyeron en equipos colaborativos 11 mapas conceptuales que se analizaron desde dos aspectos: estructura/topología y contenido semántico (Cañas et al., 2015). En la Tabla 2 se presentan las valoraciones cuantitativas para los indicadores de cada uno de estos aspectos.

Tabla 2.

Número de mapas conceptuales $(\mathrm{N})$ y porcentaje (\%) en cada nivel de la escala* para los indicadores que describen los aspectos.

\begin{tabular}{llrrc}
\hline \multicolumn{1}{c}{ Aspecto } & \multicolumn{1}{c}{ Indicadores } & Escala & $\mathbf{N}$ & $\%$ \\
\hline Topología/ & & 3 & 1 & 9,1 \\
estructura & $\begin{array}{l}\text { Uso de conceptos en vez de trozos de texto desde } \\
\text { la perspectiva estructural }\end{array}$ & 4 & 7 & 63,6 \\
\cline { 2 - 4 } & & 5 & 3 & 27,3 \\
\cline { 2 - 5 } & Establecimiento de relaciones entre conceptos & 1 & 1 & 9,1 \\
\cline { 2 - 5 } & & 2 & 2 & 18,2 \\
\cline { 2 - 5 } & & 4 & 1 & 9,1 \\
\hline
\end{tabular}




\begin{tabular}{|c|c|c|c|c|}
\hline Aspecto & Indicadores & Escala & $\mathbf{N}$ & $\%$ \\
\hline \multirow{10}{*}{$\begin{array}{l}\text { Topología/ } \\
\text { estructura }\end{array}$} & El grado de ramificación & 5 & 11 & 100 \\
\hline & La profundidad jerárquica & 1 & 1 & 9,1 \\
\hline & & 3 & 2 & 18,2 \\
\hline & & 4 & 4 & 36,4 \\
\hline & & 5 & 4 & 36,4 \\
\hline & La presencia de enlaces cruzados & 1 & 4 & 36,3 \\
\hline & & 2 & 2 & 18,2 \\
\hline & & 3 & 1 & 9,1 \\
\hline & & 4 & 2 & 18,2 \\
\hline & & 5 & 2 & 18,2 \\
\hline \multirow[t]{11}{*}{ Contenido } & Conceptos & 3 & 4 & 36,4 \\
\hline & & 4 & 4 & 36,4 \\
\hline & & 5 & 3 & 27,3 \\
\hline & Palabras de enlace o conectores & 1 & 2 & 18,2 \\
\hline & & 2 & 1 & 9,1 \\
\hline & & 4 & 6 & 54,5 \\
\hline & & 5 & 2 & 18,2 \\
\hline & Proposiciones & 2 & 2 & 18,2 \\
\hline & & 3 & 1 & 9,1 \\
\hline & & 4 & 6 & 54,5 \\
\hline & & 5 & 2 & 18,2 \\
\hline
\end{tabular}

*Sin embargo, en este apartado solo se incluyeron las escalas en las cuales se ubicaron los mapas conceptuales diseñados por los docentes en formación.

A partir de esta tabla se reconoce que en lo relativo a la estructura o topología, uno de los mapas presentados por los docentes en formación utiliza el mismo número de conceptos que explicaciones extensas (oraciones, ideas), mientras que siete incluyeron más conceptos. Un total de tres mapas conceptuales tuvieron la valoración más alta, puesto que solo incluyeron conceptos; esto es relevante porque, según Novak y Cañas (2006), se debe evitar oraciones estructuradas y complejas dentro de las cajas a manera de conceptos, ya que no permiten leer proposiciones y es fiel muestra de aprendizaje memorístico. 
Ahora bien, en lo relativo al uso de los conectores para establecer relaciones entre conceptos, un mapa conceptual no empleó ningún conector y dos utilizaron en menor proporción los conectores entre conceptos; mientras que la mayoría, es decir ochos mapas, permitieron ver conectores como mecanismo para relacionar conceptos y darle sentido al mapa.

Por otra parte, un punto de ramificación, según Cañas et al. (2006), ocurre cuando de un nodo, concepto o frase de enlace salen dos o más líneas de conexión (el número exacto no importa). En lo referente a este indicador, la totalidad de los mapas conceptuales elaborados por los participantes presentaban un grado de ramificación alto en tanto conceptos-conectores y conectores-conceptos.

En relación con el indicador de jerarquización, Cañas et al. (2006) lo definen como "el número de enlaces que hay entre el concepto raíz y el concepto más alejado del concepto raíz. Este es un criterio que sólo tiene sentido para mapas que contienen al menos un concepto raíz" (p. 5). Entre tanto, ocho de los mapas presentaron cuatro niveles o más.

Ahora bien, con respecto a los enlaces cruzados, es necesario recordar que hace referencia a la formación de círculos cerrados como circuitos, los cuales dan cuenta de la presencia de proposiciones. Las evidencias dieron cuenta de que cuatro mapas conceptuales construidos por los docentes en formación no incluyeron enlaces cruzados. El análisis de este criterio significó un aspecto desfavorable, puesto que la mayoría de los mapas conceptuales no incluyeron enlaces cruzados o incluyeron solamente un enlace. Así que no se tuvo evidencias de procesos de reconciliación integradora de calidad. En este caso, al rastrear su ausencia, se encontró que los mapas conceptuales con puntaje bajo fueron dos de los construidos en la $\mathrm{EF}_{2}$ y dos de los tres mapas construidos por los docentes en ejercicio que participaron en la $\mathrm{EF}_{3 .}$.

Estos hallazgos se relacionan con lo planteado por Pedrajas y Martínez (2016), quienes reconocen que los mapas conceptuales son una herramienta metacognitiva referenciada en las últimas décadas, soportada en la teoría de aprendizaje significativo. La divulgación de su efectividad y su uso en los procesos de formación inicial de docentes está en auge, sin embargo, no hay evidencias de su uso en la formación continua de docentes, como el caso de los participantes de la $\mathrm{EF}_{3}$.

A partir de estas ideas, en lo relativo a la formación inicial o continua de docentes, se evidenció un efectivo tránsito de lo meramente representacional al aprendizaje de conceptos, en tanto conceptos mismos y vínculos o relaciones entre estos. Sin embargo, las proposiciones que se construyen y los significados sí se pueden compartir, discutir, negociar y convenir. En particular, la construcción de los mapas conceptuales, por grupos de dos o tres docentes en formación, cumplieron una función de reconstrucción social del conocimiento sobre el cambio climático, animada por las discusiones, posicionamientos críticos y la explícita conexión de conocimientos procedentes de diferentes áreas.

\section{Las actitudes ambientales y frente al cambio climático}

Las actitudes ambientales se valoraron a través de un cuestionario de facetas adap- 
tado de Páramo y Gómez (1997). Este cuestionario medía las actitudes emocionales, cognoscitivas y conductuales sobre el medio ambiente, en general, y el cambio climático, en particular. Los resultados de la aplicación del cuestionario se presentan en la Tabla 3, donde se exponen las medias (y desviación estándar) de los diferentes aspectos actitudinales para cada una de las experiencias de formación de docentes.

Tabla 3.

Media aritmética y desviación estándar, por grupos sobre los diferentes aspectos actitudinales: emocionales, cognoscitivos y conductuales.

\begin{tabular}{lccccccc}
\hline Aspectos actitudinales & $\mathrm{EF}_{1} \mathrm{~N}=12$ & $\mathrm{EF}_{2}$ & $\mathrm{~N}=18$ & \multicolumn{2}{c}{$\mathrm{EF}_{3} \mathrm{~N}=9$} \\
\cline { 2 - 7 } & $\chi$ & $\sigma$ & $\chi$ & $\sigma$ & $\chi$ & $\sigma$ \\
\hline Emocionales & 4,5 & 0,24 & 4,1 & 0,5 & 4,1 & 0,3 \\
\hline Conductuales & 3,7 & 0,45 & 3,4 & 0,68 & 3,7 & 0,26 \\
\hline Cognoscitivos & 4,2 & 0,48 & 4,1 & 0,35 & 3,7 & 0,69 \\
\hline
\end{tabular}

Los resultados muestran que la media general de todos los grupos en los ítems del cuestionario sobre los aspectos emocionales fue de 4,3 (en una escala Likert de 1 a 5), es decir, hay compromiso emocional en todos los casos frente a ciertas cuestiones y/o problemáticas ambientales. Hay una diferencia en la desviación estándar mayor en el grupo $\mathrm{EF}_{2}$ es decir la experiencia de formación inicial de los profesores de Ciencias (Física/ Química, Biología/Geología) tiene valores más dispersos, tanto hacia los extremos positivo y negativo de la escala. Mientras que, para la $\mathrm{EF}_{1}, \mathrm{la}=0,24$ indica que los maestros en formación inicial que formaron parte de esta experiencia tuvieron puntuaciones más altas en los ítems relativos a lo emocional o afectivo sobre el medio ambiente que los participantes de las $\mathrm{EF}_{2-3}$.

En relación con las actitudes cognoscitivas, los ítems de este tipo de actitudes apuntaron a la toma de decisiones desde el uso de los conocimientos que tenían sobre el medio ambiente. A partir de estos datos, la media más baja y la desviación estándar más alta la registraron los docentes de la $\mathrm{EF}_{3}$; esto indi- ca puntuaciones más bajas y dispersas sobre el aspecto cognoscitivo de las actitudes ambientales que los docentes de la $\mathrm{EF}_{1} \mathrm{y} \mathrm{EF}_{2}$.

Finalmente, actitudes conductuales como la disposición real para el cambio de acciones puntuales a nivel personal y profesional -es decir, como docentes- obtuvo una media ligeramente más baja en la $\mathrm{EF}_{2}$.

\section{Conocimiento curricular}

Después de organizar la estructura conceptual sobre el nodo metadisciplinar relativo al cambio climático (proceso descrito anteriormente), los participantes en las $\mathrm{EF}_{1}$ 2-3 realizaron una lectura de la Ley Orgánica para la Mejora de la Calidad Educativa -LOMCE- para primaria, secundaria y bachillerato, según el caso. El primer obstáculo que se encontraron los docentes es que los temas ambientales no se encuentran en todos los casos explícitos en el currículo, lo cual generó algunos conflictos, confusión y dificultad para canalizar los procesos formativos. 
DF22: A nivel curricular, hemos considerado que se puede tratar en varios cursos, en especial en la ESO se puede tratar en primero, tercero y cuarto y en otra asignatura que es ciencias aplicadas a la actividad profesional, se puede tratar sobre todo en investigación y desarrollo o en diferentes bloques de aplicación al medio ambiente o bien al desarrollo de proyectos de investigación.

$\left(\mathrm{EF}_{2}\right.$, fase semi dirigida colaborativa. Transcripción de audio. 03/05/2016)

La aproximación al currículo permitió a los docentes en formación visibilizar una tarea complicada y aún pendiente en el contexto escolar, que implica otorgarle un significado realmente transdisciplinar a lo ambiental. Los equipos colaborativos reconocieron durante las $\mathrm{EF}_{1-2-3}$ que:

DF20: Cuando empezamos a ver el currículo de la ESO (de Conocimiento del Medio), nos ha costado mucho ver dónde encajarlo, porque es más visto a lo mejor en Tecnología o en Biología, pero en Física y Química no sabíamos muy bien cómo encajarlo. Entonces, se nos ocurrió la idea de enfocarlo hacia el tema de la combustión de la gasolina y del uso de los hidrocarburos; a partir de la combustión empezamos a desarrollar sobre ¿cómo y por qué se producía el calentamiento global?

$\left(\mathrm{EF}_{2}\right.$ fase semidirigida colaborativa. Transcripción de audio. 03/02/2016)

En relación con los elementos curriculares que tuvieron en cuenta los participantes a la hora de diseñar sus estrategias didácticas en la $\mathrm{EF}_{1}$, durante su formación inicial habían cursado una serie de asignaturas relativas al currículo, por esta razón, los elementos curriculares eran familiares y formaban parte de sus discursos. Sin embargo, las estrategias didácticas no estaban enriquecidas en todos los casos con dichos elementos. Mientras que los profesores de Ciencias en formación que hicieron parte de la $\mathrm{EF}_{2}$ cuidaron de que el nodo metadisciplinar permitiera vincular diferentes áreas de conocimiento, haciendo referencia a todos los elementos curriculares.

Por otra parte, los docentes en ejercicio presentaron al final de la $\mathrm{EF}_{3}$ un total de ocho estrategias didácticas; una mencionó las competencias y tres hicieron el esfuerzo de integrar otras áreas de conocimiento diferentes al conocimiento del medio:

DF33: Podemos incluir la materia de Ciencias Sociales, ya que hay que incluir el apartado de localización geográfica de Tudela, para relacionarla a su vez con un clima en concreto, que la va a relacionar con un paisaje característico que van a definir nuestro ecosistema. Igualmente, podríamos incluir aspectos de la materia de Tecnología, por el hecho de emplear el ordenador con diferentes programas, la materia de plástica al tener que realizar algún dibujo que se pide, como el de una seta con sus partes, la materia de Educación Física al sugerirles que paseen por la zona para identificar las plantas, imágenes, etc.

$\left(\mathrm{EF}_{3}\right.$, fase semi dirigida-colaborativa. Estrategia didáctica de una docente. Junio de 2016)

Como se ha mencionado en otros apartados, al iniciar las EF, los participantes consideraban que la transdisciplinariedad era un reto, puesto que implica una nueva forma de concebir los procesos educativos, en general, y los procesos en educación ambiental, en particular. Sin embargo, manifestaban que era relevante la voluntad administrativa (directivos y docentes) en los centros educativos. Además, aspectos como la cooperación estratégica entre los docentes también se mencionaron. 
DF24: Necesita la voluntad de hacerlo, y claro, no puede ser voluntad de una persona, tienen que ser de un grupo implicado en desarrollar un tema de modo transversal.

DF25: Se necesita mucha cooperación de los profesores de las diferentes asignaturas. A mí me parece que puede ser una buena idea.

DF31: Normalmente se suele rechazar, si no lo dominas, habíamos comentado antes que una de las ventajas que tienen los institutos pequeños es que el mismo profesor de Física y Química da Tecnología, así que en este caso la transversalidad sería más sencilla. Pero dos o tres profesores distintos es muy complicado.

$\left(\mathrm{EF}_{2}\right.$, fase mediadora. Transcripción de audio. 03/02/2016)

Estas evidencias explícitas permiten comprender que sí hay una dificultad para conectar conocimientos procedentes de diferentes disciplinas, especialmente a la hora de plantear estrategias didácticas, lo cual confirma que la interdisciplinariedad y la transdisciplinariedad es una tarea pendiente en educación ambiental.

\section{Conocimiento didáctico del contenido.}

Durante la fase semidirigida colaborativa de las EF, a los docentes en formación se les asignó un enfoque didáctico entre los que se encontraron los siguientes: modelización, aprendizaje por indagación, aprendizaje basado en problemas (APB), aprendizaje basado en el lugar/contexto, herramientas TIC, aprendizaje por descubrimiento (APD). En equipos colaborativos (2-3 personas) profundizaron sobre estos enfoques y diseñaron la estrategia didáctica teniendo en cuenta la caracterización, contextualización o aspec- tos relativos a la proyección educativa (es decir, pensando en el proceso de enseñanza y aprendizaje de sus futuros alumnos) y la secuenciación de actividades.

Durante la $\mathrm{EF}_{1}$, los docentes en formación inicial diseñaron 5 estrategias didácticas; por su parte, en la $\mathrm{EF}_{2} \mathrm{y} \mathrm{EF}_{3}$ diseñaron 17 estrategias. Puntualmente, la estrategia de modelización aportó a la reestructuración disciplinar y posibilitó pensar la forma de llevar los conocimientos de diferentes ciencias, es decir, aquellos concernientes a la Biología, Física, Química y Geología, pero también, conocimientos implicados en el principio de sustentabilidad (ingenieriles, humanistas), puesto que a través de modelos se representan diversas situaciones ambientales. Sin embargo, el uso de estos modelos da cuenta de la complejidad de las relaciones. Por su parte, durante la $\mathrm{EF}_{3}$, los docentes en ejercicio construyeron modelos más simples partiendo de la literatura, mostrando dificultades para recontextualizarlos en una situación ambiental particular como el cambio climático.

Las salidas de campo conocidas en la tradición anglosajona como outdoor activities, así como las visitas a los museos, como ambientes de aprendizaje no convencionales, posibilitaron apropiar el conocimiento y transferirlo al contexto cercano a partir de la construcción y/o uso de modelos en educación ambiental.

Por otro lado, las estrategias basadas en enfoques como el aprendizaje por indagación, el aprendizaje basado en problemas, el aprendizaje por descubrimiento y el aprendizaje basado en lugar dejan aspectos susceptibles de exponer. En primer lugar, demanda tiempo dominar los componentes 
epistemológicos y praxeológicos de cada enfoque y, por ende, llevar a cabo una planeación efectiva. En segundo lugar, tienen relevancia si se vinculan y transforman el currículo, de lo contrario serían estrategias poco viables para los docentes y los centros educativos. En tercer lugar, implican diseño de materiales y dinamización de espacios de enseñanza y aprendizaje, aspecto también condicionado por los contextos educativos. En cuarto lugar, se evidencia que la innovación en solitario es más compleja para el docente, por lo tanto, este tipo de estrategias son mucho más efectivas cuando se cuenta con pares de apoyo: otros docentes, directivos, pares académicos.
Con respecto a las TIC se concluye que, a pesar de la tendencia a incorporarlas como herramientas o instrumentos para la gestion educativa, en lo relativo a la educación ambiental, es necesario transcender y utilizar las TIC desde una perspectiva de avanzada, para responder a las problemáticas ambientales: realidad virtual, realidad aumentada, conexión a gran escala para reportar y compartir datos reales (real data) a nivel local o global. Los juegos en línea cobran cada vez más fuerza y deben ser motivadores para la formación de docentes con la intención de que conozcan, dominen y lleven al aula este tipo de herramientas.

\section{Conclusiones}

En relación con los conocimientos sobre el cambio climático, se reconoce el interés por lo político e histórico. Además, se valora el mapeo conceptual como estrategia potencialmente significativa, puesto que favoreció la reestructuración, evolución y apropiación del conocimiento sobre el tema, desde una perspectiva inter y transdisciplinar. Además, permitió la consolidación de proposiciones y la progresión hacia el pensamiento sistémico.

En cuanto a lo actitudinal en relación con el cambio climático, se concluye que los participantes tienen actitudes emocionales o afectivas favorables. Sin embargo, debe fortalecerse lo cognoscitivo para concretar acciones y la participación en iniciativas que mitiguen los problemas asociados.

En relación con la vinculación del currículo con la EA, los resultados dan cuenta de la necesidad de una mayor integración y que se encuentre la manera de transversalizar lo ambiental en respuesta a las realidades y problemáticas de la sociedad actual; este asunto todavía es un reto en el contexto iberoamericano. Se concluye que es necesario conceptualizar e investigar en relación con la generación de un currículo ambientalizado para la formación -inicial, continua y avanzada- de docentes.

Si bien, la alfabetización climática es un término de mayor tradición en contextos investigativos como Australia y Estados Unidos de América, forma parte del conjunto de nuevas alfabetizaciones (científica, ecológica, digital, ambiental, entre otras) y es necesario adaptarlo para el proceso de formación de docentes, de quienes socialmente se espera que tengan mayores conocimientos y mejores actitudes hacia el medio ambiente, además de un compromiso para abordar temáticas ambientales en el aula, como es el caso del cambio climático. 


\section{FinANCIACIón}

La investigación doctoral de la que se deriva este escrito fue financiada por el proyecto europeo de cooperación internacional Erasmus Mundus Eurica (2015-2017).

\section{Nota}

口

1. Tesis doctoral titulada Alfabetización ambiental y profesionalización docente: Diseño e iteración de un modelo de formación (Lopera-Pérez, 2019).

\section{REFERENCIAS}

Astolfi, J. P. (1988). El aprendizaje de conceptos científicos: aspectos epistemológicos, cognitivos y lingüísticos. Enseñanza de las Ciencias, 6(2), 147-155.

Azevedo, J., \& Marques, M. (2017). Climate literacy: a systematic review and model integration. International Journal of Global Warming, 12(3/4), 414-430.

Ballantyne, R. (1995). Environmental Teacher Education: Constraints, Approaches and Course Design. International Journal of Environmental Education and Information, 14(2), 115-128.

Bolívar, A. (2005). Conocimiento didáctico del contenido y didácticas específicas. Profesorado. Revista de currículum y formación del profesorado, 9(2), 1-39.

Cañas, A. J., Novak, J. D., \& Reiska, P. (2015). How good is my concept map? Am I a good Cmapper? Knowledge Management \& E-Learning: An International Journal, 7(1), 6-19.

Gaudiano, E. J. G., \& Cartea, P. Á. M. (2020). Educación para el cambio climático: ¿educar sobre el clima o para el cambio? Perfiles Educativos, 42(168), 157-174.

Gess-Newsome, J. (2015). A model of teacher professional knowledge and skill including PCK: Results of the thinking from the PCK Summit. In A. Berry, P. Friedrichsen y J. Loughran (Eds.), Re-examining Pedagogical Content Knowledge in Science Education (pp. 28-42). New York: Routledge.

Goldman, D., Yavetz, B., \& Pe'er, S. (2006). Environmental literacy in teacher training in Israel: Environmental behavior of new students. The Journal of Environmental Education, 38(1), 3-22.

Köpen, W. (1948). Climatología. Buenos Aires, Argentina: Gráfica Panamericana.

Krippendorff, K. (2004). Content analysis: An introduction to its methodology. London, UK: Sage. 
Landsberg, H.E. (1981): The urban climate. New York, USA: Academic Press.

Lopera-Pérez, M. (2019). Alfabetización ambiental y profesionalización docente: Diseño e iteración de un modelo de formación. Tesis doctoral. Universidad de Valladolid.

Nayan, N., Mahat, H., Hashim, M., Saleh, Y., \& Norkhaidi, S. B. (2018). Verification of the Instrument of Climate Literacy Knowledge among Future Teachers: Confirmatory Factor Analysis (CFA). Development, 7(3).

Niepold, F., Herring, D., \& McConville, D. (2007). The case for climate literacy in the 21st century. In 5th International Symposium on Digital Earth. from http://www. isde5. org.

Novak, J. D., y Cañas, A. J. (2006). La teoría subyacente a los mapas conceptuales ya cómo construirlos. Reporte Técnico IHMC CmapTools, 1, 1-37.

Plutzer, E., McCaffrey, M., Hannah, A. L., Rosenau, J., Berbeco, M., \& Reid, A. H. (2016). Climate confusion among US teachers. Science, 351(6274), 664-665.

Páramo, P., y Gómez, F. (1997). Actitudes hacia el medio ambiente: su medición a partir de la teoría de facetas. Revista latinoamericana de Psicología, 29(2), 243-266.

Pedrajas, A. P., y Martínez, M. V. (2016). Mapas conceptuales aplicados al tratamiento de temas medioambientales en la formación del profesorado de física. Profesorado: Revista de curriculum y formación del profesorado, 20(2), 452-472.

Reeves, T. C., Herrington, J., \& Oliver, R. (2005). Design research: A socially responsible approach to instructional technology research in higher education. Journal of Computing in Higher Education, 16(2), 96-115.

Payne, P. G. (2006). Environmental education and curriculum theory. The Journal of Environmental Education, 37(2), 25-35.

Pérez-Rodríguez, U., Varela-Losada, M., Lorenzo-Rial, M. A., y Vega-Marcote, P. (2017). Tendencias actitudinales del profesorado en formación hacia una educación ambiental transformadora. Revista de Psicodidáctica, 22(1), 60-68.

Sauvé, L. (1996). Environmental education and sustainable development: A further appraisal. Canadian Journal of Environmental Education, 1, 7-34.

Sauvé, L. (2005a). Currents in Environmental Education: Mapping a Complex and Evolving Pedagogical Field. Canadian Journal of Environmental Education, 10(1), 11-37.

Shulman, L. S. (1986). Those who understand: Knowledge growth in teaching. Educational Researcher, 15(2), 4-14.

Tejada-Fernández, J. (2009). Profesionalización docente en el escenario de la Europa de 2010. Una mirada desde la formación. Revista de educación, (349), 463-477. 
UNESCO (1994) Informe Final de la Conferencia Mundial: Necesidades Educativas Especiales. Acceso y Calidad. Madrid, España: UNESCO, Ministerio de Educación y Cultura.

UNESCO (1997). Recomendación relativa a la condición del personal docente de la enseñanza superior. París, Francia: UNESCO.

Vezub, L. (2005). Tendencias internacionales de Desarrollo Profesional Docente. La experiencia de México, Colombia, Estados Unidos y España. Trabajo realizado a solicitud de las Direcciones Nacionales de Gestión Curricular y Formación Docente y de Investigación y Evaluación de la Calidad Educativa del Ministerio de Educación, Ciencia y Tecnología de la Argentina. Buenos Aires, septiembre de 2005.

Wang, F., \& Hannafin, M. J. (2005). Design-based research and technology-enhanced learning environments. Educational technology research and development, 53(4), 5-23. 